\title{
Vertical disparity tolerance in random-dot stereograms
}

\author{
K. PRAZDNY \\ Schlumberger Palo Alto Research Center, Palo Alto, California
}

\begin{abstract}
The binocular visual system cannot process vertical disparity differences larger than about $10^{\prime}$ without using corrective eye movements.
\end{abstract}

Vertical disparities in binocular vision are the consequence of the differential distance of an object to the nodal points of the two eyes and of the (probably unavoidable) eye misalignment inherent in the biological hardware. Although vertical disparities alone do not produce a depth sensation (Ogle, 1964), they do carry, in principle at least, information about some global parameters of the binocular system, for example, the convergence angle (LonguetHiggins, 1981; Mayhew \& Longuet-Higgins, 1982). Such computations are possible because of the redundancy inherent in the requirement that the directions of gaze must be the same whatever set of corresponding points is used to "calculate" them (Prazdny, 1983).

Most current computational theories of stereomatching (Marr \& Poggio, 1976, 1979; Mayhew \& Frisby, 1980; Prazdny, 1985) are two-stage theories which assume that the actual stereomatching process responsible for detecting horizontal disparities is preceded by a coarse registration mechanism that approximately aligns the two eyes to allow the fine matching to become essentially a onedimensional search process. While there is not much known about this (still) hypothetical coarse registration mechanism, it has been suggested that it may be driven, through vergence eye movements, by various monocular cues, for example, texture, luminance, or perhaps even color or kinetic edges (Kidd, Frisby, \& Mayhew, 1979; Poggio \& Poggio, 1984; Ramachandran, Madhusudhan Rao, \& Vidyasagar, 1973).

An interesting question concerns then the maximum range of vertical disparities that allows fusion without eye movement in complex displays (e.g., random-dot stereograms) which present inherent ambiguity to the stereomatching system.

A binocular form defined by purely vertical correlations (vertical disparities) is easily perceived against the binocularly uncorrelated surround of a random-dot field. The form is always seen in front of such background, independently of the sign (up or down) of the vertical disparities. We have observed that when two so-defined objects, each with different amounts of vertical disparity,

The author's mailing address is: Artificial Intelligence Laboratory, FMC Corporation, 1185 Coleman Ave., B580, Santa Clara, CA 95052. are presented side by side, only one can be seen at any time when the vertical disparity difference is more than about $10^{\prime}$. In such situations, the vergence eye movements can apparently "correct" only for one vertical disparity setting (corresponding to one object). When the disparity difference is so large that the other object is not seen at all, its region is perceived as indistinguishable from the uncorrelated background. In other words, the stereomatching system cannot tolerate vertical disparities greater than about $10^{\prime}$. This initial observation formed the basis of a formal experiment described below.

\section{METHOD}

\section{Procedure}

The random-dot stereograms were generated on a Symbolics 3600 LISP machine and displayed on a high-resolution, noninterlaced CRT (refresh rate $60 \mathrm{~Hz}$ ) with P4 (fast) phosphor. The viewing distance was $75 \mathrm{~cm}$. From this distance, each $(260 \times 260$ pixels $)$ half-image subtended approximately $6.5^{\circ}$ (i.e., one pixel was approximately $1.5^{\prime}$ ). The target objects were two vertical bars on a binocularly uncorrelated background. Each bar was $1^{\circ}$ (40 pixels) wide. The separation between the bars was $40^{\prime}$. The stereograms were fused by crossing the eyes in front of the display. Each stereogram was presented until the subject reported seeing at least one bar. At that time, the subjects simply indicated whether one or both bars could be discerned. Every subject participated in three sessions. In two sessions, the subjects fixated between the two bars (a small fixation mark was placed between them). In the third session, the subjects fixated one of the bars. The order of the sessions was chosen at random by computer. Each session consisted of 10 upper bound measurements. Each measurement consisted of increasing or decreasing (determined by a random choice) the vertical disparity difference between the two bars by $1^{\prime}$, beginning at $0^{\prime}\left(15^{\prime}\right)$, until the subjects could not (or just could) perceive both bars simultaneously. The values from increasing and decreasing trials were averaged.

\section{Subjects}

Three observers with normal acuity and stereo vision viewed the displays. The subjects were experienced in free fusion of stereograms by crossing the eyes but naive as to the purpose of the experiment.

\section{RESULTS AND DISCUSSION}

No difference was found between the three trials (i.e., the fixation place did not play any measurable role). The results from all three trials were averaged together and are displayed in Figure 1. As a further qualification of the data, we note that no subject could perceive the two bars simultaneously for vertical disparity differences at or above $10^{\prime}$, and all subjects saw the two bars for verti- 


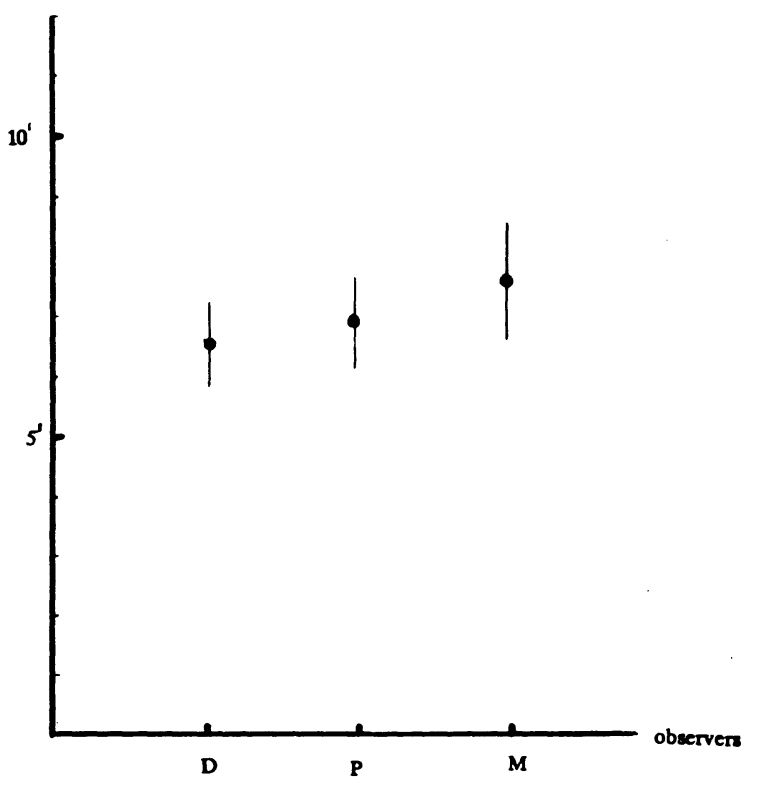

Figure 1. Maximal vertical disparity difference at which two bars can be perceived. Above these values the observers perceived only one of the bars. Each data point is the mean of $\mathbf{3 0}$ measurements. Vertical bars are standard deviations.

cal disparity differences at or below $6.5^{\prime}$. Another interesting point noted by our observers was that the edges of the two bars became "fuzzy" with increasing vertical disparity.

The results of these simple experiments support recent findings of Nielsen \& Poggio (1984) who used different, more complex procedures and reported vertical tolerances up to about $8^{\prime}$. Our results also are in good agreement with values obtained using more conventional line stimuli
(Duwaer, 1982; Duwaer \& van der Brink, 1982; Schor \& Tyler, 1981).

In short, vertical disparities tolerated by the stereomatching process are rather small, at most $10^{\prime}$, or roughly half of the horizontal extent of Panum's fusional area.

\section{REFERENCES}

Duwaer, A. L. (1982). Non-motor component of fusional response to vertical disparity. Journal of the Optical Society of America, 72, 871-877.

Duwaer, A. L., \& VAN Der Brink, G. (1982). Detection of vertical disparities. Vision Research, 22, 467-478.

KidD, A. L., Frisby, J. P., \& MAYHeW, J. E. W. (1979). Texture contours can facilitate stereopsis by initiating appropriate vergence eye movements. Nature, 280, 829-832.

LONGUET-Higgins, H. C. (1981). A computer algorithm for reconstructing a scene from two projections. Nature, 293, 4133-4135.

MARR, D., \& Poggio, T. (1976). A cooperative computation of stereo disparity. Science, 194, 283-287.

MARR, D., \& Poggio, T. (1979). A theory of human stereopsis. Proceedings of the Royal Society of London, B204, 301-328.

Mayhew, J. E. W., \& Frisby, J. P. (1980). The computation of binocular edges. Perception, 9, 69-87.

Mayhew, J. E. W., \& Longuet-Higgins, H. C. (1982). A computational model of binocular depth perception. Nature, 297, 376-378.

Nielsen, K. R., \& Poggio, T. (1984). Vertical image registration in stereopsis. Vision Research, 24, 1133-1140.

OGLE, K. N. (1964). Researches in binocular vision. New York: Hafner.

Poggio, G. F., \& Poggio, T. (1984). The analysis of stereopsis. Annual Review of Neuroscience, 7, 379-412.

PraZdNy, K. (1985). Detection of binocular disparities. Biological Cybernetics, 52, 387-395.

Prazdny, K. (1983). Stereoscopic matching, eye position, and absolute depth. Perception, 12, 151-160.

Ramachandran, V. S., Madhusudhan Rao, V., \& Vidyasagar, T. R. (1973). The role of contours in stereopsis. Nature, 242, 412-414.

SCHOR, C. M., \& TYLER, C. W. (1981). Spatio-temporal properties of Panum's fusional area. Vision Research, 21, 683-692.

(Manuscript received for publication May 20, 1985.) 